\title{
Masers: Probing the Mass Loss Process in PPN
}

\section{Dieter Engels}

\author{
Hamburger Sternwarte, Gojenbergsweg 112, D-21029 Hamburg
}

With the advent of the IRAS All-Sky Survey a sizeable number of transition objects between the AGB and the PN-phase were found - the Proto Planetary Nebulae (PPN). Oxygenrich $\mathrm{AGB}$ stars often show prominent masers of $\mathrm{SiO}, \mathrm{H}_{2} \mathrm{O}$, and $\mathrm{OH}$, which are lost during the transition process. The heavy mass loss on the AGB however does not stop abruptly and a new axisymmetric wind develops during the PPN phase. These winds both may host new masers and they can be used to study the changes of the mass loss process after that the stars have stopped their large-amplitude variations on the AGB. Several PPN are known to have $\mathrm{OH}$ masers, and at least in one case, HD 101584, the presence of a bipolar outflow could be proven (te Lintel Hekkert et al. 1992). Lewis (1989) found that main-line $\mathrm{OH}$ masers become prominent again. I will discuss here conclusions, which can be drawn from observations of $\mathrm{H}_{2} \mathrm{O}$ masers in PPN.
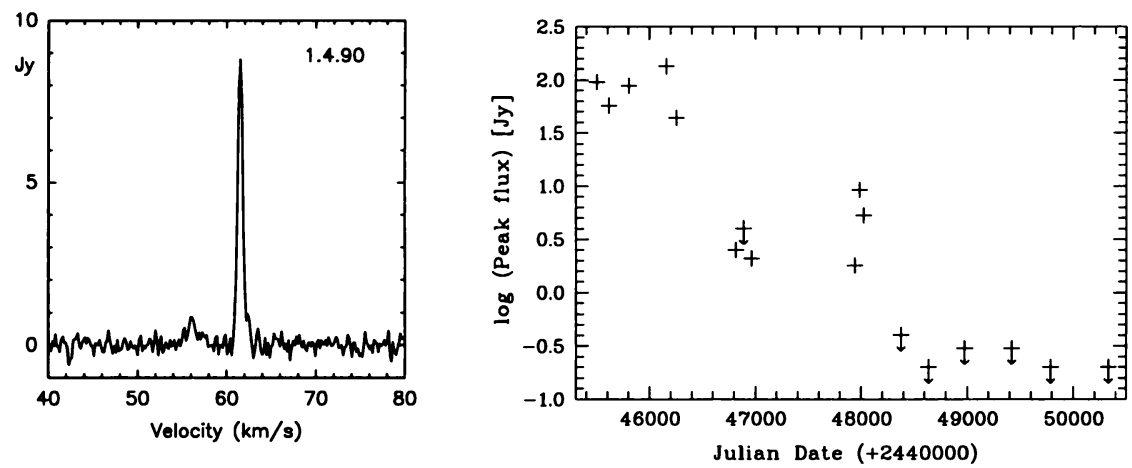

(a) Representative $\mathrm{H}_{2} \mathrm{O}$ maser spectrum of $\mathrm{OH}$ 17.7-2.0. The emission occurs close to the center of the $\mathrm{OH}$ velocity range between 47 and $75 \mathrm{~km} \mathrm{~s}^{-1}$ (Type A spectrum).

(b) Lightcurve of the maser between 1983 and 1996. For each epoch the peak flux of the strongest line was taken. Data are from Nyman et al. (1986), Engels et al. (1986), Likkel (1989) and Engels (in preparation).

I monitored the $1.3 \mathrm{~cm} \mathrm{H}_{2} \mathrm{O}$ maser emission of four "non-variable" $\mathrm{OH} / \mathrm{IR}$ stars using the $100 \mathrm{~m}$-radiotelescope in Effelsberg. The stars were selected because of their peculiar maser spectra, and their maser lightcurves cover now more than 10 years. "Nonvariable" OH/IR stars are considered as PPN because the large-amplitude pulsation has stopped and evidence was gathered from infrared measurements that they have detached circumstellar shells. Two stars, $\mathrm{OH} 15.7+0.8$ and $\mathrm{OH} 17.7-2.0$, show Type A maser spectra, e.g. the emission is concentrated at the radial velocity of the star (Figure 1a). This is unusual, because the FIR dust emission measured by IRAS and the $\mathrm{OH}$ maser emission infer mass loss rates of $>10^{-5} \mathrm{M}_{\odot} \mathrm{yr}^{-1}$ and Type A maser profiles occur on the AGB 


\section{From AGB to Planetary Nebula}

only in stars with lower mass loss rates $\left(<10^{-6} \mathrm{M}_{\odot} \mathrm{yr}^{-1}\right)$. At higher mass loss rates the maser consists of two line complexes close to the velocities of the $\mathrm{OH}$ maser peaks (Type B spectra) (Engels et al. 1986). I suggest that these masers trace the Post-AGB wind with a mass loss rate of $\approx 10^{-7} \mathrm{M}_{\odot} \mathrm{yr}^{-1}$, which is in this case simply the final phase of the decaying AGB wind. Given typical shell sizes of $\mathrm{OH}$ masers, the start of the decay of the mass loss rate occured less than 200-2000 years ago. In addition to the peculiar spectral shapes, the time evolution of their maser spectra is absolutely remarkable. The $\mathrm{H}_{2} \mathrm{O}$ maser of $\mathrm{OH}$ 17.7-2.0 has undergone a dramatic decrease in intensity since its discovery in 1983. After 1990 the maser disappeared completely (Figure 1b). From observations of semiregular and blue Mira variables we know that a threshold mass loss rate somewhere between $10^{-8}$ and $10^{-7} \mathrm{M}_{\odot} \mathrm{yr}^{-1}$ exists, below which $\mathrm{H}_{2} \mathrm{O}$ masers are not observed anymore. One is therefore tempted to attribute the decrease of $\mathrm{H}_{2} \mathrm{O}$ maser luminosity to a rapid decrease of the mass loss rate in $\mathrm{OH} 17.7-2.0$ by a factor $\approx 10$ well below a level of $10^{-7} \mathrm{M}_{\odot} \mathrm{yr}^{-1}$. By accident we may have witnessed the very short period of $\mathrm{H}_{2} \mathrm{O}$ maser emission from the AGB wind, dying out on the extremely short time scales of 20-30 years proposed by Bedijn (1987). OH 15.7+0.8 shows a similar light curve and disappeared three years ago. These $\mathrm{H}_{2} \mathrm{O}$ masers give further evidence for a smooth transition of the mass loss process at the end of the AGB evolution, a conclusion already reached before by Bedijn from modelling of the infrared energy distributions of these stars.

The two other "non-variable" OH/IR stars monitored are $\mathrm{OH}$ 12.8-0.9 and $\mathrm{OH} 37.1$ 0.8 , which show water masers outside the $\mathrm{OH}$ maser velocity range. Their intensities remained constant over the time although individual maser lines appeared and disappeared with lifetimes of $1-3$ years. Their inferred $\mathrm{H}_{2} \mathrm{O}$ outflow velocities are $\approx 11$ and $\approx 12 \mathrm{~km} \mathrm{~s}^{-1}$ higher than their $\mathrm{OH}$ outflow velocities, which is incompatible with the standard model of AGB circumstellar shells, in which the expansion velocity increases outwards. I share the opinion of Gomez et al. (1994) that these masers trace a new Post-AGB wind, possibly with bipolar geometry. These stars might be linked to the "water fountain" sources with $\mathrm{H}_{2} \mathrm{O}$ and $\mathrm{OH}$ outflow velocities of $40-130 \mathrm{~km} \mathrm{~s}^{-1}$ (Likkel et al. 1992). For OH 12.8-0.9 an increase of the $\mathrm{H}_{2} \mathrm{O}$ maser outflow velocity was observed during the monitoring program, indicating that the outflow traced by this maser is still accelerated. We may witness a star, which is currently developing the high ouflow velocities seen presently in "water fountain" sources.

The search for peculiar masers in cool IRAS sources provides a powerful tool to discover very young PPN, before they have left the main strip in the IRAS color-color diagram populated by ordinary $\mathrm{OH} / \mathrm{IR}$ stars.

\section{REFERENCES}

Bedijn P.J., 1987, A\&A 186, 136.

Engels D., Schmid-Burgk J., Walmsley C.M., 1986, A\&A 167, 129.

Gomez Y., Rodriguez L.F., Contreras M.E., Moran J.M., 1994, RMexA\&A 28, 97.

Lewis B.M., 1989, ApJ 338, 234.

Likkel L., 1989, ApJ 344, 350.

Likkel L., Morris M., Maddalena R.J., 1992, A\&A 256, 581.

Nyman L-Å., Johansson L.E.B., Booth R.S., 1986, A\&A 160, 352.

te Lintel Hekkert P., Chapman J.M., Zijlstra A.A., 1992, ApJ 390, L23. 